\title{
Therapeutic Targeting of Signal Transduction Pathways and Proteins in Breast Cancer
}

\author{
Nancy E. Hynes • William Gullick
}

Published online: 22 July 2006

(C) Springer Science + Business Media, Inc. 2006

Cancer results from cumulative alterations of the genetic make-up of somatic cells that lead to aberrant expression, mutation or deletion of proteins modulating cellular proliferation, differentiation and survival. Through deregulation of intracellular pathways, these defects allow cancer cells to evade signals, which normally keep cell proliferation and survival tightly controlled [1]. Furthermore, the tumor microenvironment facilitates processes, including angiogenesis and matrix breakdown, that are necessary for cancer cells to metastasize from the primary site to distant organs. In the past 25 years our knowledge of the genetic and epigenetic changes that contribute to cancer has increased tremendously. In contrast, we are only beginning to appreciate the complex interaction between tumor cells and their stromal environment [2]. Our knowledge of alterations in the cancer cell have, on the one hand, allowed us to identify the signaling pathways that when disrupted allow a cancer cell to escape from normal control mechanisms. On the other hand, the aberrant processes have also provided many therapeutic points for intervention, which is the major topic of the current issue. We have chosen a selection of potential molecules and pathways that are altered in breast cancer and are under scrutiny as targeted therapeutics. Some of these like the ERBB receptors have yielded therapeutics that have already had clinical success;

N. E. Hynes $(\bowtie)$

Friedrich Miescher Institute for Biomedical Research,

Basel, Switzerland

e-mail: Hynes@fmi.ch

W. Gullick

Cancer Biology Laboratory, Research School of Biosciences,

University of Kent,

Canterbury, UK

e-mail: W.J.Gullick@kent.ac.uk while others are still in the experimental stages. With our knowledge of the molecular alterations in breast cancer and the increasing number of new targeted therapeutics, socalled signal transduction inhibitors (STIs), the current challenge is to select the best combination of STIs, or an appropriate STI with a standard chemotherapy for a particular patient.

Receptor tyrosine kinases are often aberrantly activated in human cancers. In breast cancer, constitutive activation of the epidermal growth factor receptor (EGFR) and ErbB2 is often found. Aberrant activation results from overexpression, mainly due to gene amplification, kinase domain mutation and autocrine or paracrine ligand induced activation. The ERBB receptors couple to multiple signalling pathways that impact on all aspect of cancer biology. Trastuzumab, an ErbB2 specific humanized monoclonal antibody was one of the first biologicals to be approved for metastatic breast cancer treatment [3]. Recently, very exciting results from three multicenter trials testing trastuzumab in the adjuvant setting reported that addition of the antibody to standard adjuvant chemotherapy was associated with a dramatic reduction in the risk to recurrence [4]. The articles by Gullick and colleagues and by Badache and Goncalves, on EGFR and ErbB2, respectively, review the roles for these receptors in breast cancer biology and as cancer targets. Sachdev and Yee discuss the IGF receptor, another important signal transducer in breast cancer.

mTOR is a central regulator of cellular responses to multiple stimuli including amino acid availability and growth factor receptor signaling. In cells with sufficient nutrients, mTOR relays a signal to the translational machinery leading to an enhanced translation of mRNAs encoding proteins essential for cell growth and cell cycle progression [5]. In breast tumors, activating mutations in PIK3A, encoding the catalytic subunit of PI3K, or loss of PTEN, the negative 
regulator of PI3K activity, are very frequent and contribute to constitutive pathway activation and mTOR activity. The mTOR pathway and emerging opportunities for intervention are the topic of a review by Hynes and Boulay.

The STATs are a family of transcription factors that relay the interactions of cytokines and growth factors with their receptors at the cell surface to mediate changes in gene expression. In normal situations STATs are transiently activated; however, in many tumors tyrosine-phosphorylated STATs, mainly STAT3 and STAT5, can be detected, suggesting constitutive pathway activation. The review by Groner and colleagues discusses STAT functions and their potential as therapeutic targets.

The NF- $\mathrm{KB}$ pathway is triggered in response to microbial and viral infections and to pro-inflammatory cytokines. These agents activate the I $\mathrm{KB}$ kinase, which phosphoylates I $\mathrm{KB}$ allowing the liberated NF- $\mathrm{kB}$ family transcription factors to enter the nucleus. The pathway has an ever increasing interest for its role in human cancer (reviewed in [6]). The association of NF-KB pathway activity with inflammation-associated tumor progression is well documented in mouse tumor models [7] and in human cancer cells [8]. Doppler and colleagues discuss the NF-KB pathway in the context of breast cancer, describing potential approaches to block the pathway in tumors.

The Notch network in mammals consists of four Notch receptors and five Delta-like and Jagged ligands. Once activated, the receptor undergoes intramembrane proteolysis leading to release and nuclear translocation of the intracellular domain, which has transcriptional activity. The Notch-4 gene is frequently rearranged in mammary glands by MMTV proviral integration. This rearrangement leads to expression of the intracellular domain under MMTV promoter control and to mammary cancer [9], an early demonstration of Notch's ability to induce mammary cancer. In this issue Shi and Harris discuss the role of Notch signaling in promotion of tumor angiogenesis.

\section{References}

1. Hanahan D, Weinberg RA. The hallmarks of cancer. Cell 2000;100:57-70.

2. Condeelis J, Pollard JW. Macrophages: obligate partners for tumor cell migration, invasion, and metastasis. Cell 2006;124:263-6.

3. Slamon DJ, Leyland-Jones B, Shak S, Fuchs H, Paton V, Bajamonde A, et al. Use of chemotherapy plus a monoclonal antibody against HER2 for metastatic breast cancer that overexpresses HER2. N Engl J Med 2001;344:783-92.

4. Piccart-Gebhart MJ, Procter M, Leyland-Jones B, Goldhirsch A, Untch M, Smith I, et al. Trastuzumab after adjuvant chemotherapy in HER2-positive breast cancer. N Engl J Med 2005;353:1659-72.

5. Wullschleger S, Loewith R, Hall MN. TOR signaling in growth and metabolism. Cell 2006;124:471-84.

6. Karin M, Cao Y, Greten FR, Li ZW. NF-kappaB in cancer: from innocent bystander to major culprit. Nat Rev Cancer 2002;2:301-10.

7. Greten FR, Eckmann L, Greten TF, Park JM, Li ZW, Egan LJ, et al. IKKbeta links inflammation and tumorigenesis in a mouse model of colitis-associated cancer. Cell 2004;118:285-96.

8. Wiener Z, Ontsouka EC, Jakob S, Torgler R, Falus A, Mueller C, et al. Synergistic induction of the Fas (CD95) ligand promoter by Max and NFkappaB in human non-small lung cancer cells. Exp Cell Res 2004;299:227-35.

9. Robbins J, Blondel BJ, Gallahan D, Callahan R. Mouse mammary tumor gene int-3: a member of the notch gene family transforms mammary epithelial cells. J Virol 1992;66:2594-99. 